\title{
Expanding shells of shocked neutral hydrogen around compact $\mathrm{H}$ ॥ regions
}

\author{
R. Kothes ${ }^{1,2}$ and C. R. Kerton ${ }^{1}$ \\ ${ }^{1}$ National Research Council of Canada, Herzberg Institute of Astrophysics, Dominion Radio Astrophysical Observatory, \\ PO Box 248, Penticton, British Columbia, V2A 6K3, Canada \\ 2 Department of Physics and Astronomy, The University of Calgary, 2500 University Dr. NW, Calgary, AB, \\ T2N 1N4 Canada \\ e-mail: charles.kerton@nrc.ca
}

Received 20 February 2002 / Accepted 30 April 2002

\begin{abstract}
By comparing radial velocities of radio bright compact $\mathrm{H}_{\text {II }}$ regions with their $\mathrm{H}_{\mathrm{I}}$ absorption profiles, we discovered expanding shells of neutral hydrogen around them. These shells are revealed by absorption of the radio continuum emission from the $\mathrm{H}$ in regions at velocities indicating greater distances than the observed radial velocity. We believe that these shells are shock zones at the outer edge of the expanding ionized region. Additionally we found evidence for a velocity inversion inside the Perseus arm caused by a spiral shock, which results in a deep absorption line in the spectra of compact $\mathrm{H}_{\text {II }}$ regions behind it.
\end{abstract}

Key words. ISM: circumstellar matter - ISM: H II regions - ISM: kinematics and dynamics

\section{Introduction}

Models of the photodissociation of molecular clouds surrounding newly formed $\mathrm{O}$ and early B type stars (Hill \& Hollenbach 1978; Roger \& Dewdney 1992) show that the resulting H I will exist in a layered structure surrounding the star. A dissociation front (DF) will first rapidly move through the molecular cloud forming a broad $\mathrm{H}_{\mathrm{I}}$ region. As the $\mathrm{H}_{\mathrm{II}}$ region expands a layer of shocked $\mathrm{H}_{\mathrm{I}}$ will form just outside the ionization front (IF). Eventually the faster moving IF will catch up with the DF and all of the $\mathrm{H}_{\mathrm{I}}$ will be found in the shocked layer. The time when the IF and DF merge ranges from $\sim 10^{5}-10^{6}$ years depending upon the spectral type of the star and the density of the surrounding molecular material (see Fig. 7 of Roger \& Dewdney 1992). The broad H I region has been detected in 21-cm line emission in a number of studies (e.g. Roger 1982), but observational evidence for the shocked layer is currently limited to a study of the H II region Orion A by van der Werf \& Goss (1989).

In this paper we present a novel technique for the detection and study of expanding $\mathrm{H}_{\mathrm{I}}$ structures around compact $\mathrm{H}_{\text {II }}$ regions that uses $21-\mathrm{cm} \mathrm{H}$ I absorption spectra. The technique allows $\mathrm{H}_{\mathrm{I}}$ surrounding $\mathrm{H}_{\text {II }}$ regions to be studied even if the $\mathrm{H}_{\text {II }}$ region is not resolved. In a number of cases we detect absorption features that are associated with shocked expanding layers of photodissociated gas. We focus on compact $\mathrm{H}$ in regions within the observed area of the Canadian Galactic Plane Survey

Send offprint requests to: $\mathrm{R}$. Kothes,

e-mail: roland.kothes@nrc.ca
$(\mathrm{CGPS})^{1}\left(74.2^{\circ} \leq l \leq 147.3^{\circ} ;-3.6^{\circ} \leq b \leq 5.6^{\circ}\right)$. The CGPS is part of the current international effort to map the Galactic plane in the 21-cm Hi line at $\sim 1^{\prime}$ resolution (Taylor 1999; McClure-Griffiths et al. 2001). At this resolution many Galactic $\mathrm{H}$ II regions will remain unresolved and the surrounding neutral material must be studied via absorption. The technique presented in this paper, applied to such regions, will greatly increase the number of $\mathrm{H}$ II regions studied using 21-cm absorption spectra.

$\mathrm{H}_{\text {I }}$ absorption spectra towards radio bright Galactic $\mathrm{H}$ in regions have been used primarily to probe the properties of the neutral ISM along the line of sight to the $\mathrm{H}$ II region (Wendker \& Wrigge 1996; Normandeau 1999). In these studies absorption features at velocities associated with the $\mathrm{H}_{\text {II }}$ region itself are usually ignored. Another application has been in Galactic structure studies; Kuchar \& Bania (1994) and Kuchar \& Bania (1990) have shown how 21-cm absorption spectra can resolve the distance ambiguity towards inner galaxy $\mathrm{H}_{\text {II }}$ regions.

The $\mathrm{H}_{\mathrm{I}}$ absorption spectrum towards a galactic $\mathrm{H}_{\text {II }}$ region can also be used to study the kinematics and structure of neutral material associated with the $\mathrm{H}_{\text {II }}$ region. Such absorption studies have tended to be limited to a few very bright $\mathrm{H}$ II regions such as DR 21 (Roberts et al. 1997), Orion (van der Werf \& Goss 1989) and W3 (van der Werf \& Goss 1990). More recently Lebrón et al. (2001) used H I absorption as part of a multiwavelength study of the blister $\mathrm{H}$ II region G111.61+0.37.

\footnotetext{
${ }^{1}$ CGPS data are publicly available via the Canadian Astronomy Data Centre (CADC; http: //cadcwww.hia.nrc.ca).
} 
In Sect. 2 we describe the data used and outline the data processing techniques. Particularly we focus on a new spatial structure filtering technique. In Sect. 3 we show the absorption spectra of all our $\mathrm{H}$ in regions. A general model for the structure of an absorption spectrum is presented and applied to our observations in Sect. 4 followed by a general discussion in Sect. 5. Finally, a summary is presented in Sect. 6.

\section{Observations and data analysis}

\subsection{HI line data from the CGPS}

$\mathrm{H}_{\mathrm{I}}$ 21-cm line data were obtained using the DRAO synthesis telescope (Landecker et al. 2000) as part of the Canadian Galactic Plane Survey (CGPS) (Taylor 1999). The H I data reduction techniques used in the CGPS are described in detail in Higgs (2000). The CGPS consists of data cubes with a size of $5.12^{\circ} \times 5.12^{\circ}$. For further analysis we extracted $1.4^{\circ} \times 1.4^{\circ}$ fields for each $\mathrm{H}$ II region studied.

\subsection{Data analysis}

To determine $\mathrm{H}_{\mathrm{I}}$ emission and absorption profiles, we have to separate the Galactic background emission from the small scale structures around and on the compact $\mathrm{H}_{\text {II }}$ regions we plan to study. For that purpose we used the "background filtering technique" invented by Sofue \& Reich (1979). This method was developed for separating smooth Galactic background emission from small scale emission structures. We have modified the technique to be useful for negative features as well as for positive ones.

For each velocity channel we used the following procedure:

$-T_{\mathrm{B}} \rightarrow T_{\mathrm{B}}^{0}$ convolution to a beam $\Theta$

$-T_{\mathrm{B}}-T_{\mathrm{B}}^{0} \rightarrow \delta T_{\mathrm{B}}$

$-T_{\mathrm{B}} \times \delta T_{\mathrm{B}} \rightarrow \Pi T_{\mathrm{B}}$

$-T_{\mathrm{B}}^{1}=\left\{\begin{array}{ll}T_{\mathrm{B}}: & \Pi T_{\mathrm{B}}<0 \\ \delta T_{\mathrm{B}}: & \Pi T_{\mathrm{B}} \geq 0\end{array}\right\}$

- $T_{\mathrm{B}}^{1} \rightarrow T_{\text {large }}$ convolution to $\Theta$

$-T_{\mathrm{B}}-T_{\text {large }} \rightarrow T_{\text {small }}$.

Here $T_{\mathrm{B}}$ is the original brightness temperature distribution with subtracted continuum emission, $T_{\text {large }}$ represents the large scale background emission, and $T_{\text {small }}$ the small scale structures including the absorption of the compact $\mathrm{H}_{\text {II }}$ regions.

For our $\mathrm{H}_{\mathrm{I}}$ data this technique creates one $\mathrm{H}_{\mathrm{I}}$ data cube representing large scale structures which provides information about the emission of the smooth background component for each source. We also get one $\mathrm{H}_{\mathrm{I}}$ data cube containing small scale structures. Absorption profiles towards the compact $\mathrm{H}$ II regions and emission profiles of possible $\mathrm{H}_{\mathrm{I}}$ structures around them are derived from this cube.

\section{Absorption profiles of compact $\mathrm{H} \|$ regions}

\subsection{The source sample}

We obtained absorption profiles towards all $\mathrm{H}$ II regions in the CGPS survey area which are unresolved in the $1420 \mathrm{MHz}$ continuum data and have a flux density greater than $200 \mathrm{mJy}$ $\left(T_{\mathrm{b}} \sim 30 \mathrm{~K}\right)$ therein. We obtained radial velocities for these $\mathrm{H}$ II regions from various sources as listed in Table 1. Sources with positive radial velocities were removed from the sample because of the distance ambiguity in the inner Galaxy. All sources in our sample are bright enough in the CGPS to show significant absorption by foreground neutral hydrogen.

\subsection{Obtained absorption profiles}

We used the background filtering technique described in Sect. 2.2 to separate the broad structures from the compact absorption features. We used a beam $\Theta$ of $5^{\prime}$ for all sources and checked all final data cubes carefully by eye. For three stronger sources, G75.77, G75.83, and G80.94, there were still some faint features from the absorption visible in the background component. For these we used a beam of $10^{\prime}$ which then successfully separated small scale and large scale structures. We created the absorption spectra in Figs. 1 to 5 by simply plotting the value at the peak position of the radio continuum source in each $\mathrm{H}_{\mathrm{I}}$ channel of the $\mathrm{H}_{\mathrm{I}}$ cubes as a function of the radial velocity. One naively expects to see absorption of the $\mathrm{H}_{\text {II }}$ region's continuum emission by the neutral hydrogen in the foreground up to its radial velocity maybe with a Gaussian slope with a width of a few $\mathrm{km} \mathrm{s}^{-1}$ towards higher negative velocities, assuming a flat rotation model. In contrast we see at least one additional absorption line well beyond the radial velocity in all but three of the 26 observed $\mathrm{H}_{\text {II }}$ regions. The additional absorption lines are at higher negative velocity than the radial velocity of the source itself indicating material which is moving towards us relative to the $\mathrm{H}_{\text {II }}$ region. Considering the fact that we find this feature in almost all of the observed compact $\mathrm{H}_{\text {II }}$ regions, we can assume that it is a symmetric expanding shell of neutral hydrogen. Since we are dealing with compact $\mathrm{H}_{\text {II }}$ regions which are unresolved in our data we do not find those expanding shells around them in emission because the emission is buried together with the foreground absorption within the beam.

We should note at this point that the additional absorption line could also be caused by a double valued rotation curve. Our sample was chosen to avoid the velocity ambiguity in the inner Galaxy, but there is also evidence for a velocity inversion in the Perseus arm caused by a spiral shock (Roberts 1972). However, since we observe this phenomenon for almost all of the compact $\mathrm{H}_{\text {II }}$ regions independent of the location and distance we assume this to be a minor factor. Nevertheless, we will discuss this in more detail in Sect. 5.

\section{Theory and modelling}

\subsection{Dissociated molecular gas around massive young stars}

In this section we summarize some of the basic results of the models of Hill \& Hollenbach (1978) and Roger \& Dewdney (1992) regarding the morphology, velocity, temperature and column density structure of the dissociated gas surrounding a 
Table 1. Characteristics of the compact $\mathrm{H}_{\text {II }}$ regions in our sample. $T_{\mathrm{c}}^{\mathrm{e}}$ represents the peak continuum brightness temperature in the CGPS $1420 \mathrm{MHz}$ data and $v_{\mathrm{LSR}}$ the radial velocity. References for the radial velocities: (1) Lockman (1989), (2) Bronfman et al. (1996), (3) Brand \& Blitz (1993), (4) Wouterloot \& Brand (1989), (5) Brunt \& Kerton (2002). Reference (1) contains recombination line velocities and all other references radial velocities of molecular lines.

\begin{tabular}{|c|c|c|c|c|}
\hline Name & $\begin{array}{c}T_{\mathrm{c}}^{\mathrm{e}} \\
{[\mathrm{K}]}\end{array}$ & $\begin{array}{r}v_{\mathrm{LSR}} \\
{\left[\mathrm{km} \mathrm{s}^{-1}\right]}\end{array}$ & Reference & other names \\
\hline G75.77+0.35 & $450 \pm 23$ & -8.5 & (1) & \\
\hline $\mathrm{G} 75.83+0.40$ & $750 \pm 38$ & -4.1 & (1) & Sh 105 \\
\hline G76.15-0.29 & $100 \pm 8$ & -28.2 & (1) & \\
\hline G76.19+0.10 & $55 \pm 6$ & -2.1 & (2) & IRAS $20220+3728$ \\
\hline G80.35+0.73 & $100 \pm 11$ & -62.4 & (1) & \\
\hline G80.94-0.13 & $374 \pm 21$ & -2.1 & (1) & IRAS $20375+4109$ \\
\hline G83.94+0.77 & $143 \pm 9$ & -8.5 & (1) & \\
\hline G85.25+0.02 & $69 \pm 7$ & -31.2 & (1) & \\
\hline G85.69+2.03 & $41 \pm 5$ & -75.0 & (4) & IRAS $20446+4613$ \\
\hline G90.24+1.72 & $32 \pm 5$ & -60.9 & (3) & Sh 121 \\
\hline G92.69+3.08 & $48 \pm 6$ & -6.4 & (2) & IRAS $21078+5211$ \\
\hline G93.86+1.56 & $44 \pm 5$ & -59.5 & (2) & IRAS $21202+5157$ \\
\hline G96.29+2.60 & $52 \pm 5$ & -94.7 & (3) & Sh 127 \\
\hline G97.51+3.17 & $72 \pm 5$ & -71.1 & (2) & Sh 128 \\
\hline $\mathrm{G} 105.62+0.34$ & $71 \pm 5$ & -52.1 & (2) & Sh 138 \\
\hline G108.20+0.58 & $259 \pm 14$ & -55.9 & (1) & Sh 146 \\
\hline G108.37-1.06 & $58 \pm 5$ & -53.1 & (3) & Sh 149 \\
\hline G108.76-0.95 & $150 \pm 9$ & -49.1 & (1) & Sh 152 \\
\hline G110.11+0.05 & $225 \pm 12$ & -51.7 & (1) & Sh 156 \\
\hline G111.62+0.37 & $87 \pm 6$ & -63.4 & (1) & Sh 159 \\
\hline G112.23+0.24 & $82 \pm 7$ & -41.8 & (1) & Sh 162 \\
\hline G114.02-1.45 & $63 \pm 5$ & -49.0 & (5) & IRAS $23365+5953$ \\
\hline G115.79-1.58 & $33 \pm 4$ & -40.8 & (1) & Sh 168 \\
\hline G132.16-0.73 & $79 \pm 5$ & -56.6 & (1) & IRAS $02044+6031$ \\
\hline $\mathrm{G} 137.75+3.80$ & $77 \pm 5$ & -47.0 & (5) & IRAS $03030+6229$ \\
\hline G138.49+1.64 & $61 \pm 5$ & -34.6 & (1) & Sh 201 \\
\hline
\end{tabular}

newly formed OB star. Essentially these models demonstrate that the dissociated gas will have a layered structure consisting of a broad unshocked layer formed by a dissociation front (DF) and a thinner shocked layer of $\mathrm{H}_{\mathrm{I}}$ located just outside of the expanding ionization front (IF).

The shocked layer of $\mathrm{H}_{\mathrm{I}}$ initially expands with the $\mathrm{H}$ II region at velocities $\sim 10 \mathrm{~km} \mathrm{~s}^{-1}$. For extremely young systems, where there has been little or no expansion of the H II region, the shocked layer of Hi will not be present (e.g. Dewdney \& Roger 1982). By the time IF merges with the more slowly moving DF the expansion velocity of the shocked layer will have dropped to $\sim 3-4 \mathrm{~km} \mathrm{~s}^{-1}$. The merging of the two fronts occurs on a time scale of $10^{5}-10^{6}$ years depending upon the density of the molecular material and the spectral type of the exciting star. The unshocked H I layer is not expected to have any systematic velocity shift relative to the molecular material if it is fully confined by the molecular material. Observations that do show expansion of the unshocked dissociated $\mathrm{H}$ I are all asso- ciated with blister H II regions (Roger 1982; van der Werf \& Goss 1989). The observed expansion velocities of $2-6 \mathrm{~km} \mathrm{~s}^{-1}$ in these cases are most likely due to the expansion of the heated dissociated gas into the surrounding low density medium.

Gas temperatures are expected to be $\leq 100 \mathrm{~K}$ within the unshocked material; newly shocked material may be hotter but is expected to cool rapidly to $T=$ few $\times 100 \mathrm{~K}$ because of efficient cooling in the shocked layer (Roger \& Dewdney 1992). Observational support for these values come from ISO observations of $\mathrm{H}_{2}$ lines towards photodissociation regions around S 140 (Timmermann et al. 1996) and S 106 (van den Ancker et al. 2000) which indicate $T \sim 500 \mathrm{~K}$, and from $\mathrm{H}_{\text {I }} 21-\mathrm{cm}$ line emission observations of a compact $\mathrm{H}$ II region in the nebular complex GGD 12-15 which indicate $T \sim 300 \mathrm{~K}$ (Gómez et al. 1998).

The column density of shocked $\mathrm{H}_{\mathrm{I}}$ is expected to grow as the system evolves, and has typical values of $10^{20}-10^{21}$ over the lifetime of the system (Roger \& Dewdney 1992). 

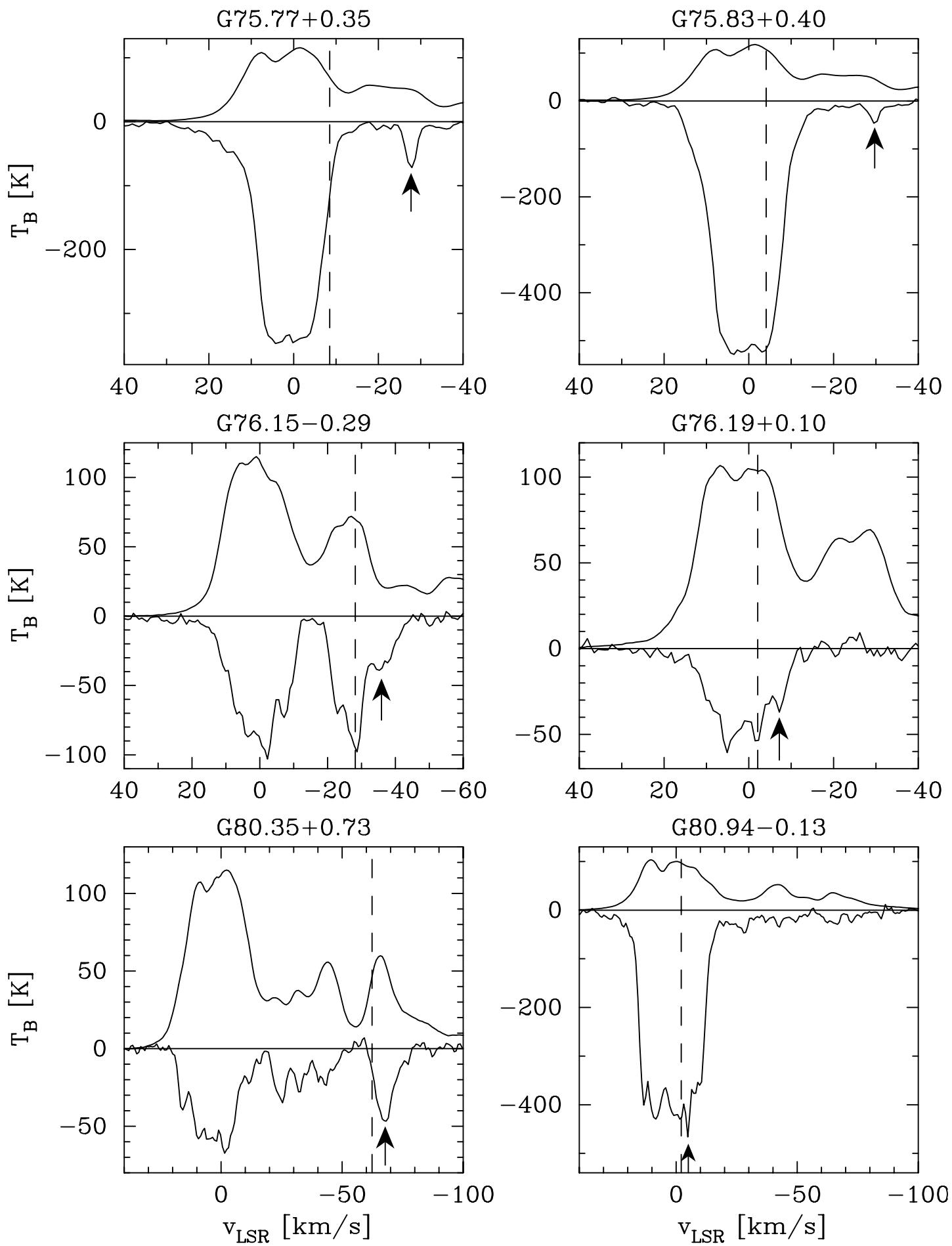

Fig. 1. $\mathrm{H}_{\mathrm{I}}$ absorption spectra of six compact $\mathrm{H}_{\text {II }}$ regions. These spectra were taken at the peak position of the continuum source. Radial velocities are indicated by a dashed line. Upper lines show the emission profile at the position of the $\mathrm{H}_{\text {II }}$ regions taken from $T_{\text {large }}$ and the lower line the absorption profile taken from $T_{\text {small }}$. Arrows indicate the absorption features of interest.

The unshocked layer is expected to reach a peak column density of $\sim 1-3 \times 10^{21}$ with little dependence on the spectral type of the exciting star and the density of the molecular material (Roger \& Dewdney 1992).

\subsection{A simple model for expanding HI shells}

To calculate observational properties of the expanding $\mathrm{HI}_{\mathrm{I}}$ shells around the compact $\mathrm{H}_{\text {II }}$ regions we have developed a simple model based upon Hill \& Hollenbach (1978) and 

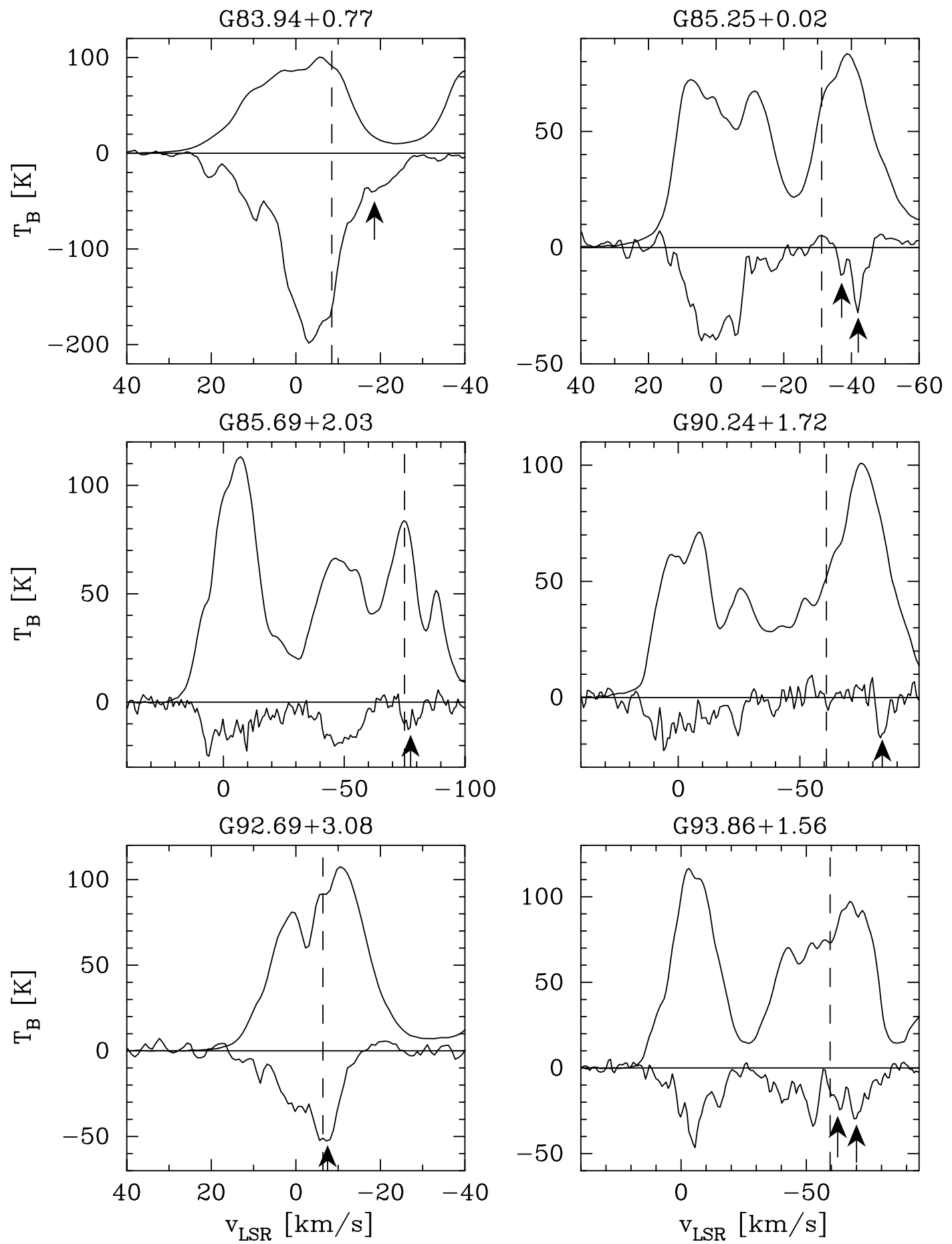

Fig. 2. see Fig. 1.

Roger \& Dewdney (1992). An early type star, located in the centre of a molecular cloud, creates an expanding $\mathrm{H}_{\text {II }}$ region inside an area of dissociated molecular material (see Fig. 6). At the interface we have a narrow zone of shocked neutral material expanding together with the ionization front. For simplicity we assume the whole structure to be symmetric. The object is located at a distance $d$ from the Sun which translates to a radial velocity $v_{\mathrm{d}}$ assuming a flat rotation curve with $v_{\odot}=220 \mathrm{~km} \mathrm{~s}^{-1}$ and $R_{\odot}=8.5 \mathrm{kpc}$. We further assume that the object is located in the outer Galaxy so that the velocity $v_{\mathrm{d}}$ is negative and a higher negative velocity implies a greater distance from the Sun. The part of the shocked Hi shell moving towards us would be shifted in its radial velocity to $v_{\mathrm{d}}-v_{\exp }$, with $v_{\exp }$ representing the expansion velocity of the $\mathrm{H}_{\mathrm{I}}$ shell, while the velocity of the receding shell would be shifted to $v_{\mathrm{d}}+v_{\exp }$ (see Fig. 6). In this case the receding $\mathrm{HI}_{\mathrm{I}}$, even though it is located behind the $\mathrm{H}_{\text {II }}$ region, would be observed among Galactic $\mathrm{H}_{\mathrm{I}}$ located between the object and the Sun. The approaching part 

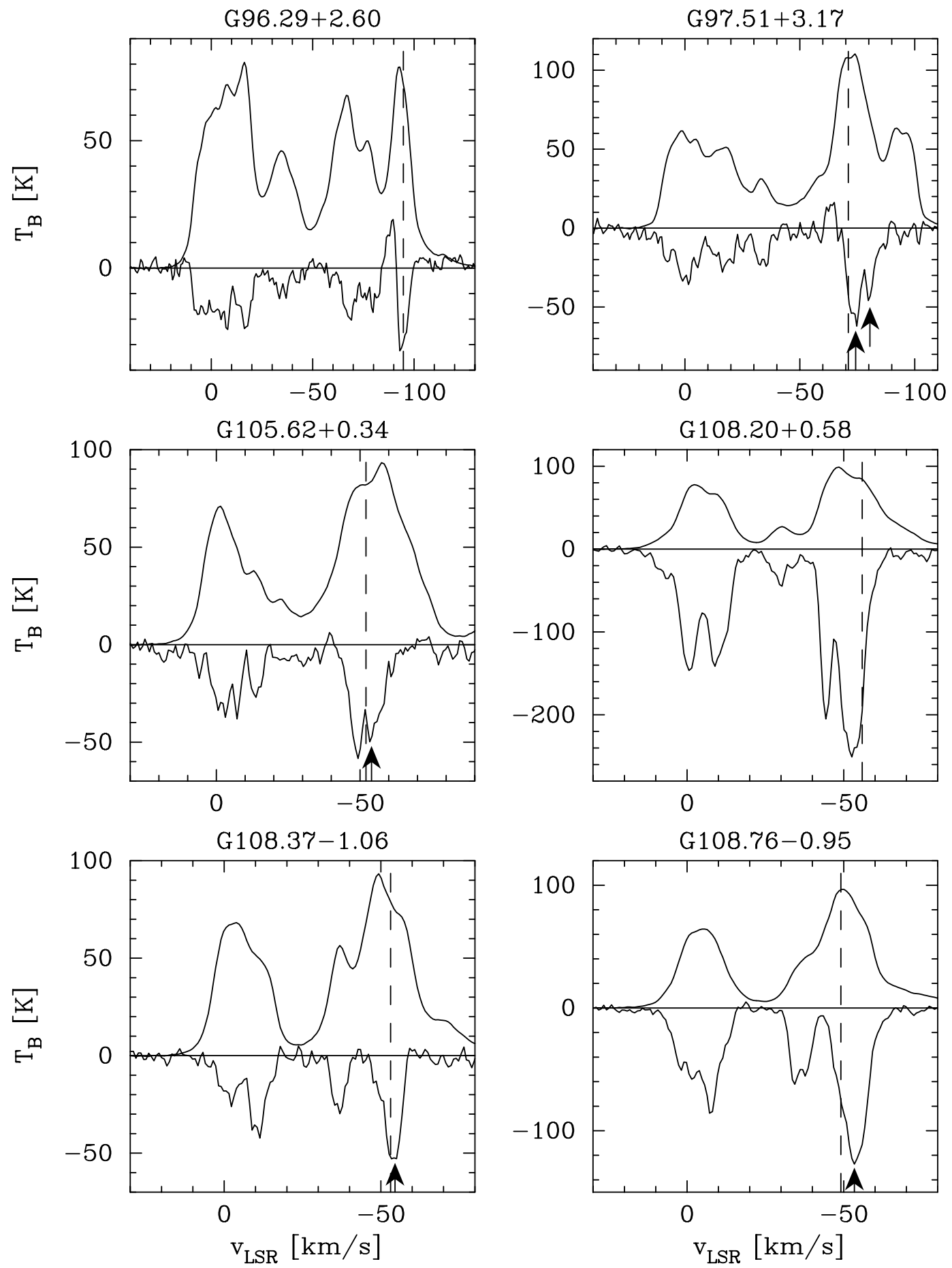

Fig. 3. see Fig. 1, positive structures in the absorption profiles of G96.29 and G97.51 are filaments coincidentally at this position and not related to the $\mathrm{H}$ in regions.

of the shell, even though located in front of the $\mathrm{H}$ II region, would appear among the material which is located behind the object in our H I data (see Fig. 6).

Assuming a constant spin temperature $T_{\mathrm{S}}$ within the shocked neutral material the observed brightness temperature $T_{\mathrm{B}}$ at the position of the $\mathrm{H}$ II region would be:

$T_{\mathrm{B}}(v)=T_{\mathrm{S}}^{\mathrm{e}}\left(1-\mathrm{e}^{-\tau(v)}\right)$ for the receding part and:

$T_{\mathrm{B}}(v)=T_{\mathrm{S}}^{\mathrm{e}}\left(1-\mathrm{e}^{-\tau(v)}\right)+T_{\mathrm{C}}^{\mathrm{e}}\left(\mathrm{e}^{-\tau(v)}-1\right)$

for the approaching part, where the second term represents the absorption of the H II region's continuum emission by the approaching $\mathrm{H}$ I shell. Here $T_{\mathrm{C}}^{\mathrm{e}}$ is the $\mathrm{H}$ II region's effective continuum brightness temperature in our $1420 \mathrm{MHz}$ data and $\tau$ is the optical depth within the H I shell. $T_{\mathrm{S}}^{\mathrm{e}}$ is the effective spin tem(1) perature. Since these compact $\mathrm{H}$ II regions are unresolved in our 

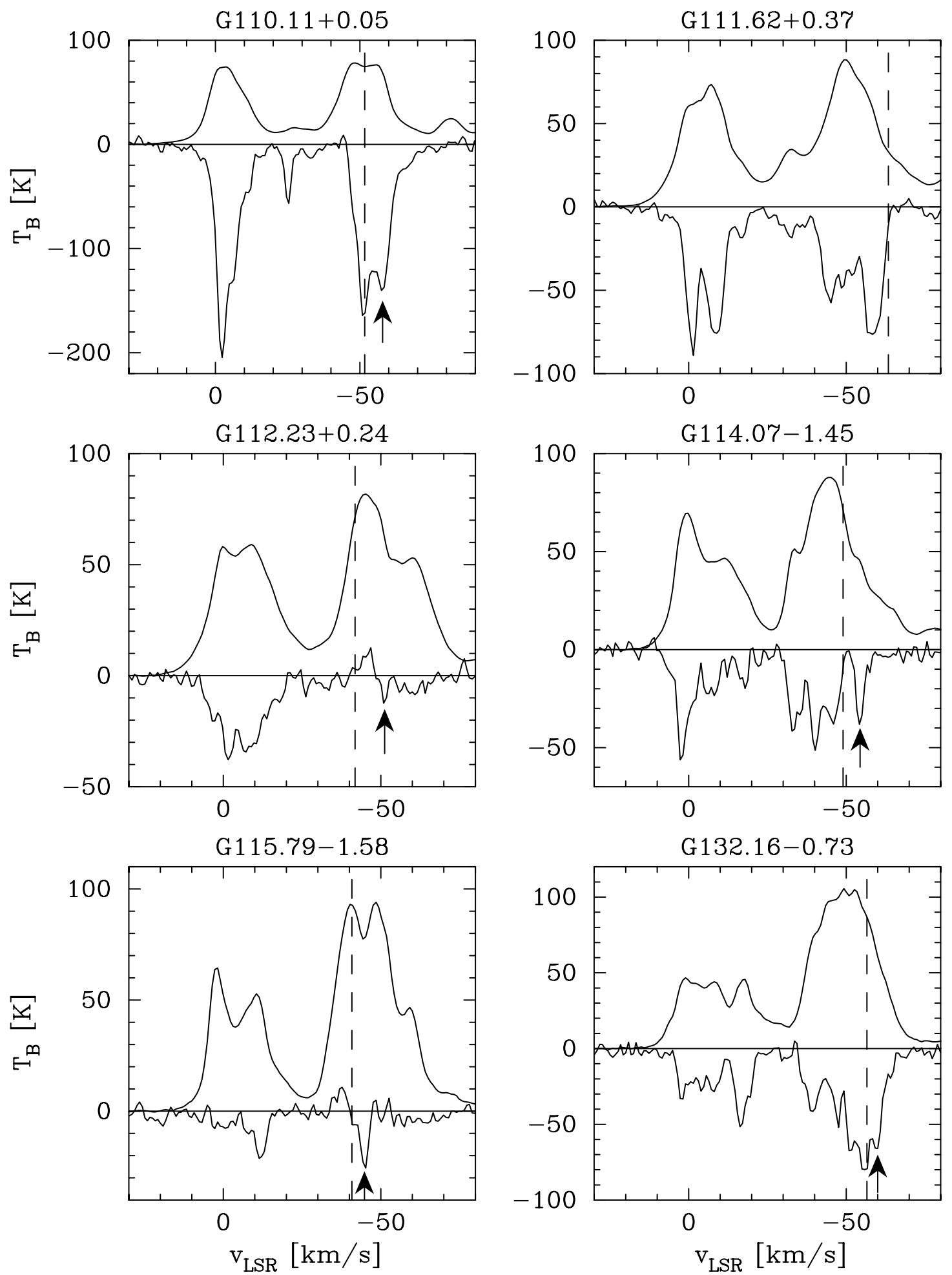

Fig. 4. see Fig. 1, positive structures in the absorption profiles of G112.23 and G115.79 are filaments coincidentally at this position and not related to the $\mathrm{H}_{\text {II }}$ regions.

data the peak brightness temperature is not the real brightness temperature of the source. $T_{\mathrm{C}}^{\mathrm{e}}$ is $T_{\mathrm{C}}$ multiplied by the ratio of the actual source area to the observed source area, that is:

$T_{\mathrm{C}}^{\mathrm{e}}=T_{\mathrm{C}} \frac{\Theta_{\mathrm{S}}^{2}}{\Theta_{\mathrm{B}}^{2}+\Theta_{\mathrm{S}}^{2}}$, where $\Theta_{\mathrm{B}}$ is the HPBW of the observation and $\Theta_{\mathrm{S}}$ the angular diameter of the source. For a source with a diameter of $10^{\prime \prime}$ and a brightness temperature of $1000 \mathrm{~K}$ for example we would measure a peak of only $30 \mathrm{~K}$. The same effect applies to the spin temperature. 

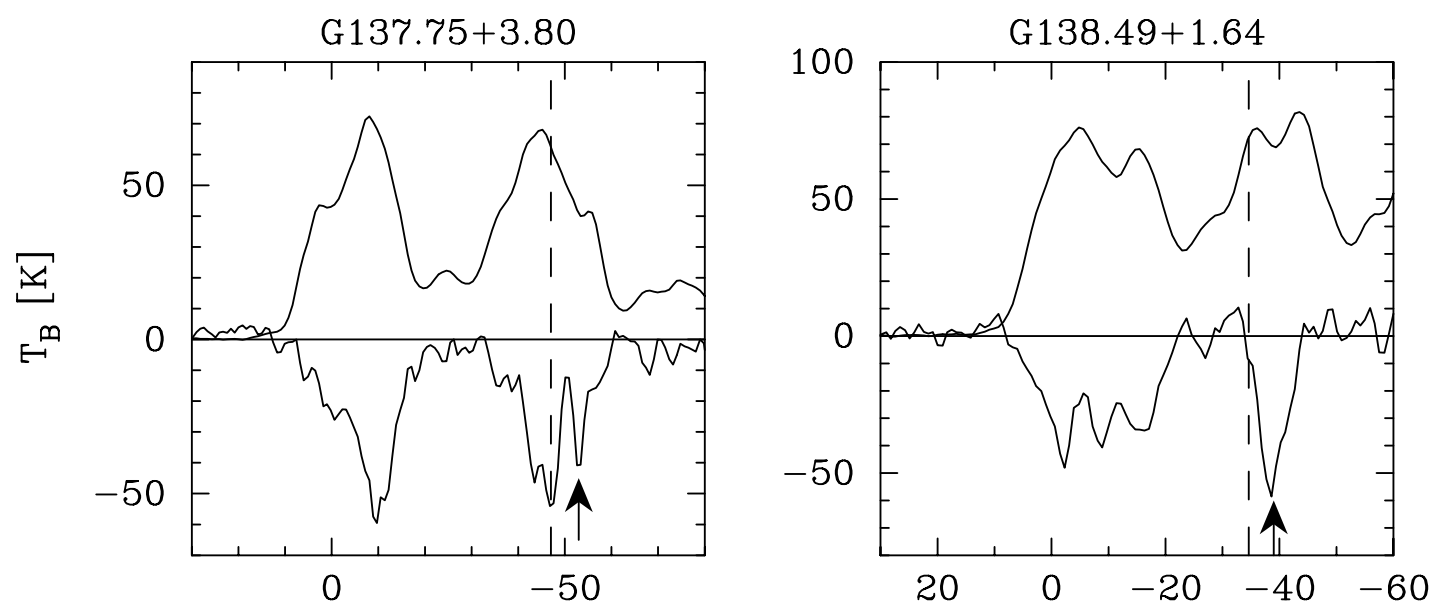

Fig. 5. see Fig. 1.
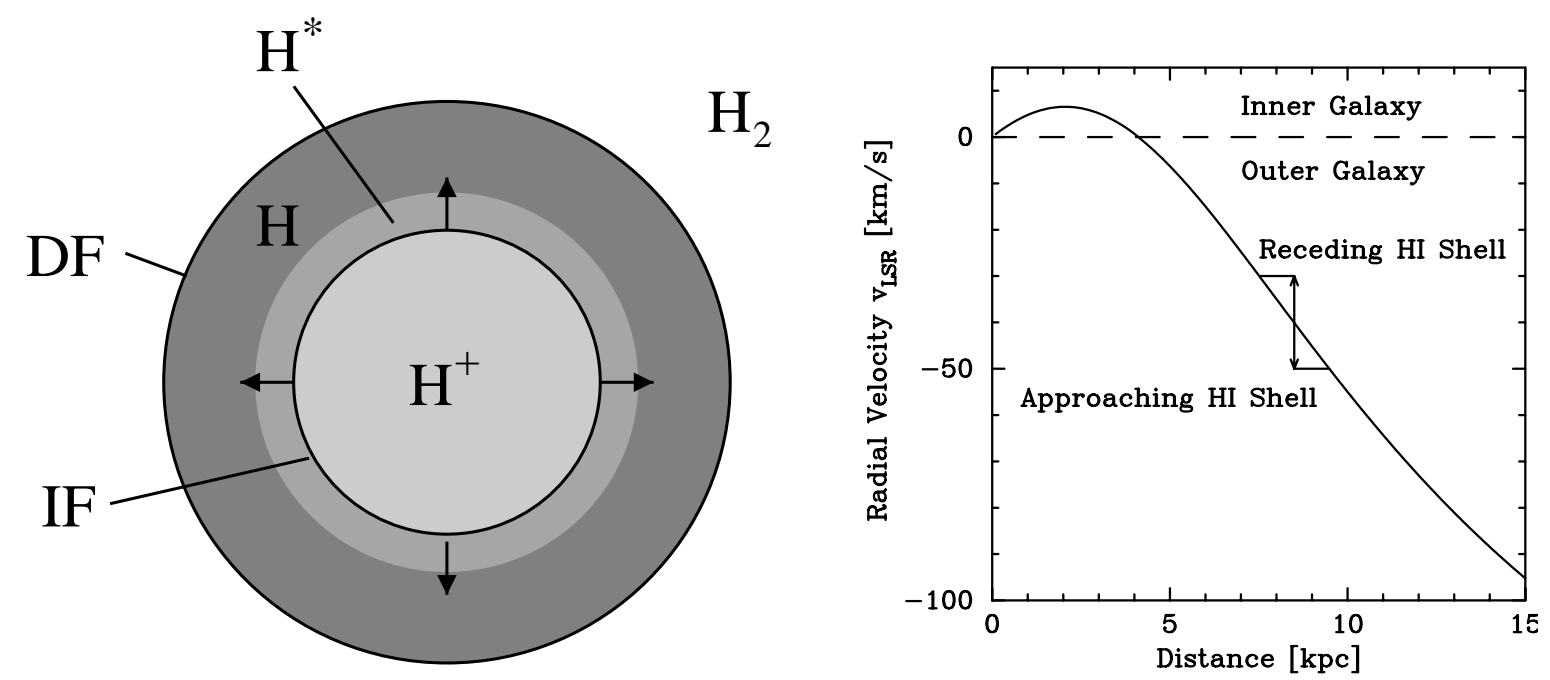

Fig. 6. Left: a sketch of a compact $\mathrm{H}_{\text {II }}$ region $\left(\mathrm{H}^{+}\right)$with an expanding layer of shocked neutral hydrogen $\left(\mathrm{H}^{*}\right)$ as described in Sect. 4.1. The dissociation front (DF) and ionization front (IF) are indicated, as is the layer of the unshocked $\mathrm{H}_{\mathrm{I}}(\mathrm{H})$. Right: a diagram of the radial velocity as a function of the distance from the Sun at a longitude of $76^{\circ}$ for a flat rotation model with $v_{\odot}=220 \mathrm{~km} \mathrm{~s}^{-1}$ and $R_{\odot}=8.5 \mathrm{kpc}$. Velocity shifts of an expanding shell with $v_{\mathrm{exp}}=10 \mathrm{~km} \mathrm{~s}^{-1}$ for an $\mathrm{H}$ II region with a radial velocity of $v_{\mathrm{d}}=-40 \mathrm{~km} \mathrm{~s}^{-1}$ are indicated.

If we now create $\mathrm{H}$ I absorption line spectra as described in Sect. 3.2 we will find continuum absorption of the $\mathrm{H}$ II regions radio emission by the foreground material up to its radial velocity. Since we find the receding part of the expanding H I shell at a radial velocity of $v_{\mathrm{d}}+v_{\text {exp }}$ it will appear as an emission line superimposed on the absorption structure of the foreground material making it hard to detect. The approaching part, however, has a radial velocity of $v_{\mathrm{d}}-v_{\exp }$. We expect to find its related line separated from the foreground absorption at a higher negative velocity.

Since our sample consists of sources which are significantly smaller than the beam of the observations, we can only speculate about the structure of neutral material around these compact $\mathrm{H}_{\text {II }}$ regions. We can resolve different layers with different expansion velocities but we do not get any information about the spatial distribution. Our method favours the part of the expanding shells which sees most of the H II region's radio continuum emission. In this case small high density knots are smoothed out and thus not detectable.

\subsection{Application to the data}

We can now calculate characteristics of the expanding $\mathrm{H}$ I shells by fitting Gaussians to the individual absorption spectra. Four examples are shown in Fig. 7. A comparison of the H II region's radial velocity with the position of the additional absorption line gives the expansion velocity of the $\mathrm{H}$ i shell. By transforming Eq. (2) we obtain an expression for the opacity $\tau$ within the expanding shell:

$\tau=-\ln \left(\frac{T_{\mathrm{B}}}{T_{\mathrm{C}}^{\mathrm{e}}-T_{\mathrm{S}}^{\mathrm{e}}}+1\right)$. 

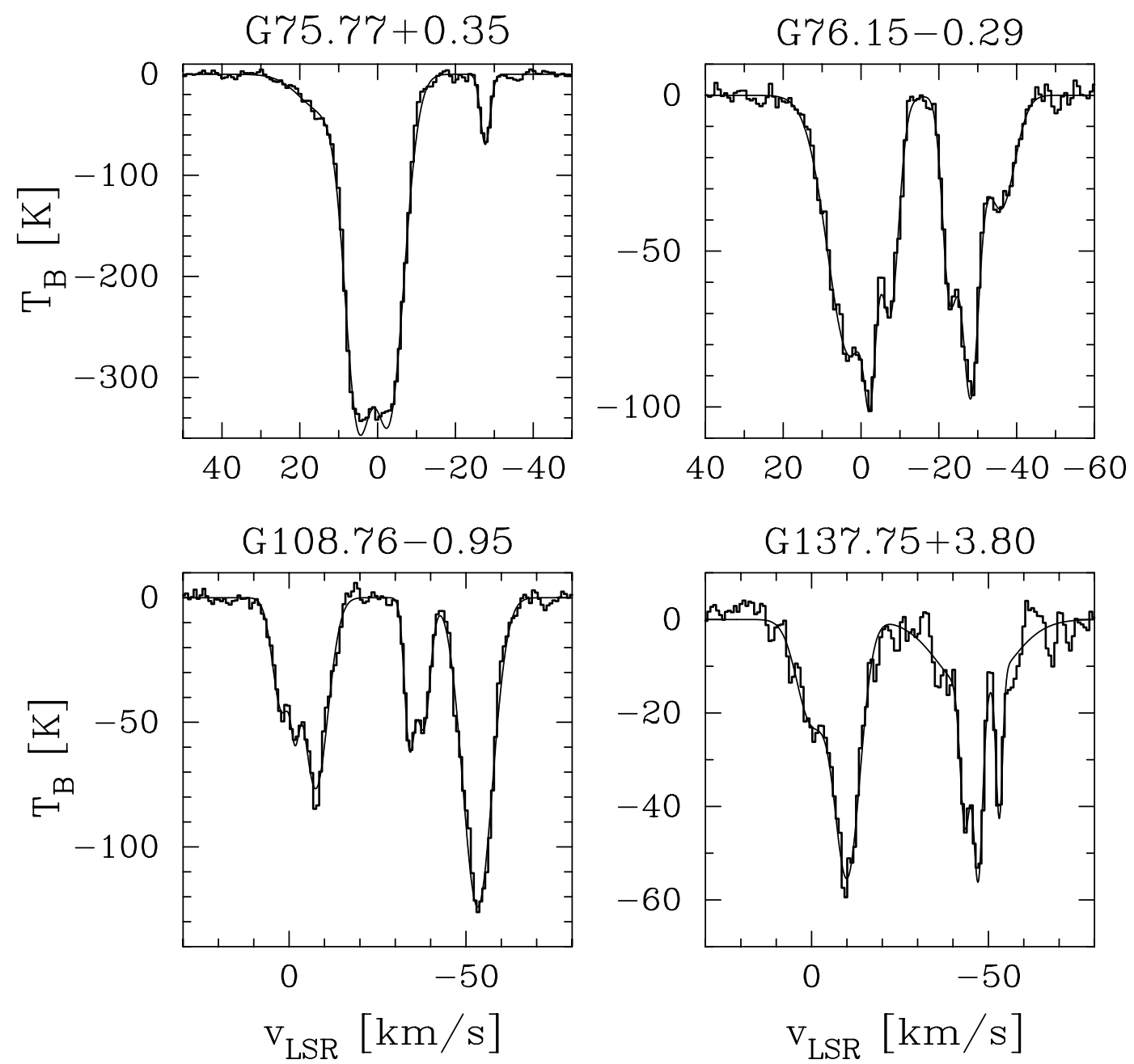

Fig. 7. Four example absorption profiles with the fitted Gaussians. The histograms represent the observed brightness temperature distribution and the solid line the fitted Gaussians.

We do not have any information about the spin temperature within the expanding $\mathrm{H}_{\mathrm{I}}$ shell since its emission is superimposed on the absorption profile. Assuming that the spin temperature is significantly lower than the continuum brightness temperature of the $\mathrm{H}$ II region we can neglect it in Eq. (3) and calculate a value for $\tau$. We should note at this point that the effect of $T_{\mathrm{S}}$ on the resulting value of $\tau$ increases with high $T_{\mathrm{B}}$ to $T_{\mathrm{C}}^{\mathrm{e}}$ ratios. As an example we also calculated the resulting $\tau$ when the spin temperature is $20 \%$ of the continuum temperature. The results for the individual $\mathrm{H}_{\text {II }}$ regions are listed in Table 2. For $\tau>2.0$ we have indicated a lower limit since the quantity $\frac{T_{\mathrm{B}}}{T_{\mathrm{C}}^{\mathrm{e}}-T_{\mathrm{S}}^{e}}$ is smaller than our uncertainties in this case. Three of the sources do not have additional absorption lines at higher negative velocities and we could not obtain a satisfactory multiple Gaussian fit to the absorption profile of G80.94 due to its complex structure.

\section{Discussion}

\subsection{The nature of the additional absorption line(s)}

The discussion about the evolution of the layer of shocked neutral hydrogen expanding with the ionizing shock front around young stellar objects in Sect. 4.1 gives us constraints on the characteristics of these features. We find a maximum expansion velocity of about $10 \mathrm{~km} \mathrm{~s}^{-1}$. An upper limit of about 1.0 for the opacity $\tau$ can be deduced from the maximum $\mathrm{HI}$ column density, $N_{\mathrm{H}}=10^{21} \mathrm{~cm}^{-2}$, and the typical spin temperature of a few $100 \mathrm{~K}$ by using the relation:

$N_{\mathrm{HI}}=1.823 \times 10^{18} \cdot T_{\mathrm{S}} \cdot \int \tau(v) \mathrm{d} v$

with

$\int \tau(v) \mathrm{d} v=1.06 \cdot \tau \cdot \delta v$

for a Gaussian distribution. Here $\delta v$ is the HPBW of the absorption line, which usually lies around a few $\mathrm{km} \mathrm{s}^{-1}$ and $\tau$ is the opacity at the peak of the line as calculated in Table 2. For an examination of those constraints within our data we plotted the opacity $\tau$ as a function of the expansion velocity for all available sources in Fig. 8. For those sources where we found double structures we plotted both results to allow for multiple lines of different origin. We found three well separated groups of $\tau-v_{\text {exp }}$ pairs in the diagram. Most sources are located in the lower left corner. Their $\tau$ and $v_{\exp }$ values agree very well with 
Table 2. Derived parameters for the compact H II regions in our sample. Sources with two additional absorption lines are noted twice.

\begin{tabular}{|c|c|c|c|c|}
\hline Name & $\begin{array}{c}v_{\exp } \\
{\left[\mathrm{km} \mathrm{s}^{-1}\right]}\end{array}$ & $\tau$ & $\begin{array}{c}\tau \\
T_{\mathrm{s}} / T_{\mathrm{c}}=0.2\end{array}$ & $\begin{array}{l}\text { HPBW } \\
{\left[\mathrm{km} \mathrm{s}^{-1}\right]}\end{array}$ \\
\hline G75.77+0.35 & $19.2 \pm 0.6$ & $0.17 \pm 0.01$ & 0.21 & 1.7 \\
\hline $\mathrm{G} 75.83+0.40$ & $25.6 \pm 0.7$ & $0.05 \pm 0.01$ & 0.07 & 1.7 \\
\hline G76.15-0.29 & $7.7 \pm 3.2$ & $0.45_{-0.05}^{+0.06}$ & 0.61 & 4.9 \\
\hline G76.19+0.10 & $5.1 \pm 0.8$ & $0.79_{-0.13}^{+0.17}$ & 1.15 & 2.8 \\
\hline G80.35+0.73 & $5.4 \pm 2.9$ & $0.64_{-0.10}^{+0.13}$ & 0.89 & 5.1 \\
\hline G80.94-0.13 & $2.7 ?$ & - & - & - \\
\hline G83.94+0.77 & $11.1 \pm 2.2$ & $0.28_{-0.02}^{+0.03}$ & 0.37 & 5.6 \\
\hline G85.25+0.02 & $10.8 \pm 2.4$ & $0.49_{-0.06}^{+0.08}$ & 0.66 & 2.4 \\
\hline G85.25+0.02 & $5.9 \pm 2.4$ & $0.23 \pm 0.03$ & 0.30 & 1.2 \\
\hline G85.69+2.03 & $2.5 \pm 0.5$ & $0.26_{-0.04}^{+0.05}$ & 0.34 & 3.8 \\
\hline G90.24+1.72 & $23.0 \pm 0.5$ & $0.91_{-0.20}^{+0.33}$ & 1.37 & 2.4 \\
\hline G92.69+3.08 & $0.8 \pm 0.8$ & $>2.00$ & $>2.00$ & 4.2 \\
\hline G93.86+1.56 & $11.0 \pm 0.8$ & $0.82_{-0.14}^{+0.19}$ & 1.21 & 6.8 \\
\hline G93.86+1.56 & $2.2 \pm 0.8$ & $0.46_{-0.07}^{+0.08}$ & 0.62 & 2.9 \\
\hline G96.29+2.60 & - & - & - & - \\
\hline G97.51+3.17 & $9.4 \pm 0.8$ & $0.70_{-0.08}^{+0.10}$ & 1.00 & 4.2 \\
\hline G97.51+3.17 & $2.2 \pm 0.8$ & $1.60_{-0.29}^{+0.41}$ & $>2.00$ & 3.5 \\
\hline G105.62+0.34 & $4.0 \pm 0.8$ & $0.28 \pm 0.03$ & 0.36 & 1.2 \\
\hline G105.62+0.34 & $1.5 \pm 0.8$ & $0.68_{-0.08}^{+0.09}$ & 0.97 & 4.2 \\
\hline G108.20+0.58 & - & - & - & - \\
\hline G108.37-1.06 & $0.6 \pm 1.3$ & $>2.00$ & $>2.00$ & 3.6 \\
\hline G108.76-0.95 & $4.3 \pm 0.9$ & $1.76_{-0.32}^{+0.46}$ & $>2.00$ & 5.8 \\
\hline G110.11+0.05 & $6.2 \pm 1.2$ & $0.78_{-0.08}^{+0.09}$ & 1.13 & 3.2 \\
\hline G111.62+0.37 & - & - & - & - \\
\hline $\mathrm{G} 112.23+0.24$ & $9.4 \pm 1.5$ & $0.16 \pm 0.02$ & 0.21 & 1.0 \\
\hline G114.02-1.45 & $5.4 \pm 0.5$ & $0.89_{-0.12}^{+0.15}$ & 1.33 & 1.7 \\
\hline G115.79-1.58 & $4.0 \pm 1.5$ & $1.46_{-0.34}^{+0.63}$ & $>2.00$ & 1.6 \\
\hline G132.16-0.73 & $2.5 \pm 4.8$ & $1.54_{-0.26}^{+0.35}$ & $>2.00$ & 3.7 \\
\hline $\mathrm{G} 137.75+3.80$ & $6.1 \pm 0.5$ & $0.51_{-0.05}^{+0.06}$ & 0.70 & 1.3 \\
\hline G138.49+1.64 & $4.3 \pm 1.0$ & $>2.00$ & $>2.00$ & 3.1 \\
\hline
\end{tabular}

the constraints we laid out for the expanding layers of shocked H I. There seems to be a sharp upper limit for the expansion velocity at about $11 \mathrm{~km} \mathrm{~s}^{-1}$ and an upper limit for $\tau$ of about 1.0 .

There is another group of sources with low $v_{\exp }$ and very high $\tau$. We should note at this point again that the $\tau$ values in this diagram were calculated by assuming that $T_{\mathrm{S}} \ll T_{\mathrm{C}}$. The effect of $T_{\mathrm{S}}$ on the final $\tau$ is bigger for $\tau$ values which are already high by assuming $T_{\mathrm{S}} \ll T_{\mathrm{C}}$ (see Table 2 ). Hence the sources located in the lower left corner are not that much affected by higher $T_{\mathrm{S}}$ values than those which are already high above this area. This means that we cannot explain the sources located high above $\tau=1$ with expanding layers of shocked $\mathrm{H}$ I.

We believe that the absorption features we are detecting in the second group indicate a possible distance ambiguity in the Perseus spiral arm. According to Roberts (1972) the spiral shock causes the radial velocity to drop by $20-30 \mathrm{~km} \mathrm{~s}^{-1}$ at the beginning of the Perseus arm and slowly rejoins the flat rotation curve after about $1 \mathrm{kpc}$. This creates a velocity inversion inside the Perseus arm. Thus inside the arm the distance is increasing with increasing radial velocity as opposed to the flat rotation curve. According to Roberts (1972) the density in the closer part of the Perseus arm is significantly enhanced by the spiral shock which would explain the high $\tau$ values we obtained. For this interpretation we would expect a strong dependence of the radial velocity of this absorption line on Galactic longitude. Because of the changing viewing angle to the Perseus arm the radial velocity should increase with increasing Galactic longitude. To examine this dependence we plotted the $v_{\mathrm{LSR}}$ at the center of the absorption line as a function of Galactic longitude, for the sources with high opacities in Fig. 9 (filled stars). 


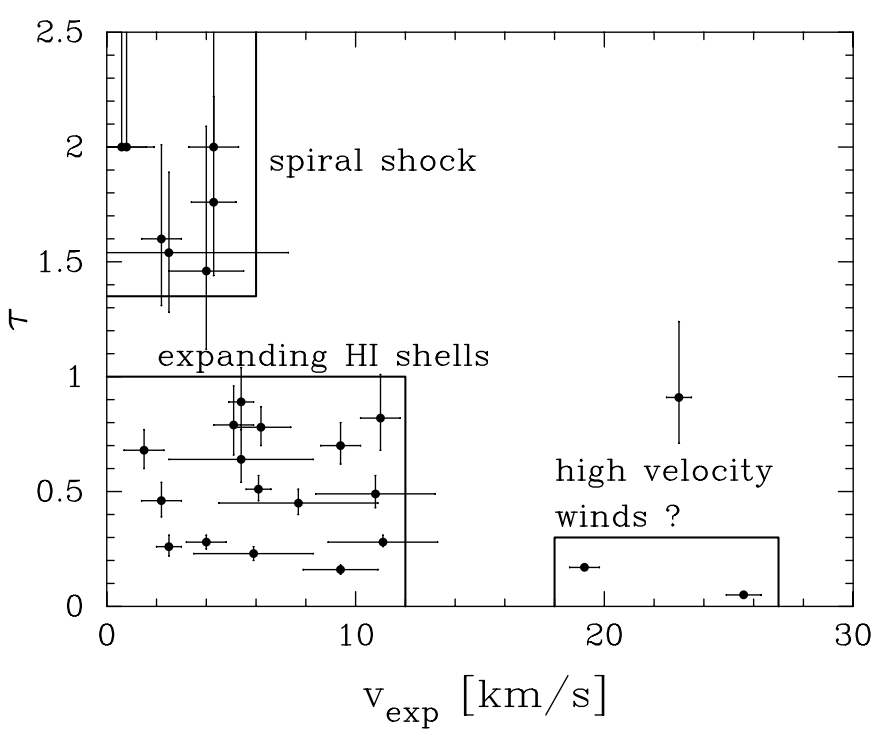

Fig. 8. Diagram with calulated minimum $\tau$ values for all $\mathrm{H}_{\text {II }}$ regions as a function of the expansion velocity. Three groups of sources with possible different origin for the additional absorption line are marked.

We added the last absorption lines from the spectra of the three sources which have no additional line at higher negative velocities and the source G90.23, one of the high velocity sources in the right part of Fig. 8 (open stars). We believe this source belongs to this group as well, because for a high velocity wind the $\tau$ is too big, and for the dissociated material, shocked or unshocked, the expansion velocity would be too high.

Apparently there is a strong relation between the radial velocities and the Galactic longitude. There are only two sources which do not follow this relation. One of those exceptions is the source G92.68 located close to the upper left corner in Fig. 9, which is of course local based on the low negative radial velocity and should not show the spiral shock in the Perseus arm at all. It has one of the lowest expansion velocities $\left(0.8 \mathrm{~km} \mathrm{~s}^{-1}\right)$ which is comparable to the uncertainty in our data. So this strong absorption line is most likely due to a dense $\mathrm{H}$ I cloud in the foreground. The second exception is the source G132.16 in the lower right area of Fig. 9. The $\tau$ is too high to be explained by the expanding shocked neutral hydrogen layer, but as mentioned in Sect. 4.1, there are rare occasions where the shell of dissociated material outside the shock front has a systematic velocity of $2-6 \mathrm{~km} \mathrm{~s}^{-1}$ for blister $\mathrm{H}$ II regions. These shells have higher Hi column densities and lower spin temperatures so we would expect higher opacities. Additionally G132.16 shows another strong absorption line in its spectrum at about $-40 \mathrm{~km} \mathrm{~s}^{-1}$ which would fit nicely to the spiral shock model.

There are 4 sources in our sample which show two additional absorption lines in their spectra instead of one. G97.51 shows one line with the characteristics of the spiral shock and the expansion velocity of the shocked neutral hydrogen is apparently high enough so that both absorption lines are well separated. The sources G85.25, G93.86, and G105.62 all have one high and one low velocity component both of which have rather low opacities. We believe that the higher velocity component is

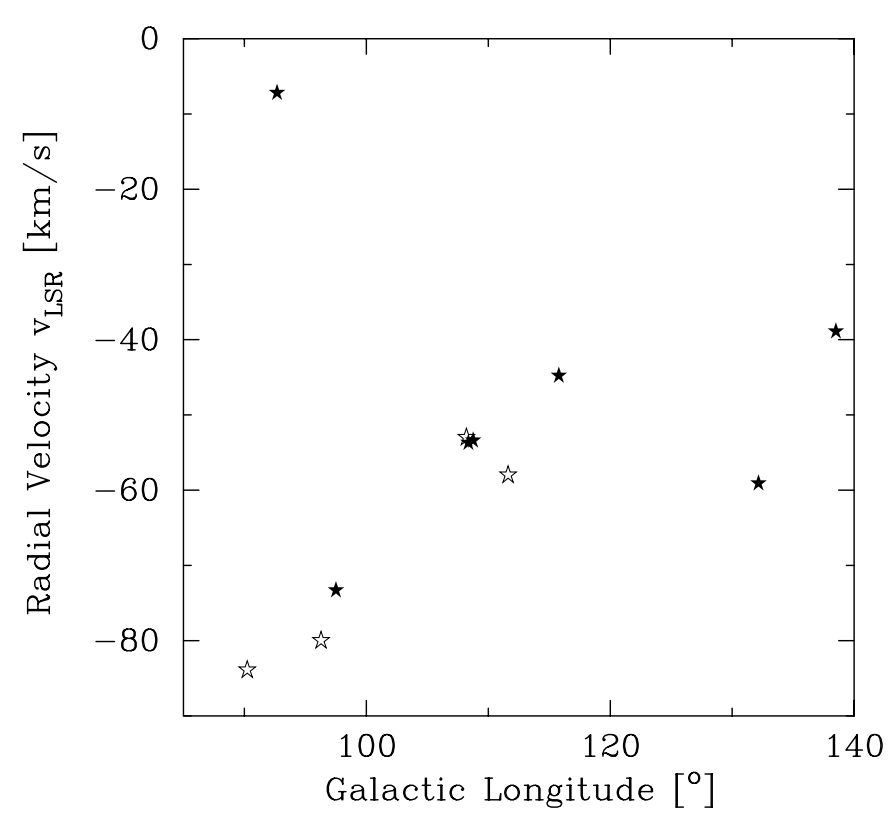

Fig. 9. Diagram of the radial velocity as a function of Galactic longitude for sources with high calculated $\tau$ values. Filled stars represent sources which are located in the box marked "spiral shocks" in Fig. 8. Open stars represent the last absorption line of those sources which have no additional absorption line at a velocity $v<v_{\text {LSR }}$ and the source G90.24.

the result of absorption by the shocked neutral hydrogen while the component with the lower velocity was produced by the unshocked hydrogen. This would indicate that all three are blister $\mathrm{H}$ II regions.

Finally, two of the observed $\mathrm{H}$ II regions, G75.77+0.35 and $\mathrm{G} 75.83+0.40$, have a very high $v_{\exp }$ and low $\tau$. Since both of these sources are very close together on the sky (only $5^{\prime}$ apart), one possibility is that we are seeing unassociated blueshifted foreground Galactic gas. We think this is unlikely though since the observed absorption line is seen at different velocities in each case $\left(v_{\mathrm{rad}}=-27.7 \mathrm{~km} \mathrm{~s}^{-1}\right.$ for $\mathrm{G} 75.77$ and $v_{\text {rad }}=-29.7 \mathrm{~km} \mathrm{~s}^{-1}$ for G75.83). Instead we believe we are observing a fast neutral outflow from these objects, perhaps formed from a recombining ionized wind. These two objects are by far the most energetic $\mathrm{H}$ II regions in our sample, and previous ${ }^{12} \mathrm{CO}$ observations have detected high velocity molecular gas, with $v \sim 10-50 \mathrm{~km} \mathrm{~s}^{-1}$, towards these objects (Shepherd \& Churchwell 1996). Models of magnetic fields in H II regions suggest that for certain magnetic field alignments fast neutral winds $\left(v \geq 20 \mathrm{~km} \mathrm{~s}^{-1}\right)$ can form from recombining ionized flows that have broken out of the molecular cloud (Franco et al. 1989). In addition, a fast neutral outflow with $v \leq 100 \mathrm{~km} \mathrm{~s}^{-1}$ has been observed in the high-mass star forming region DR 21 (Russell et al. 1992). From this, we conclude that we are seeing in absorption the blueshifted part of a fast neutral outflow coming from these objects although the linewidth is surprisingly narrow in both cases. The velocity shift in this case is the outflow velocity projected along the line of sight. 


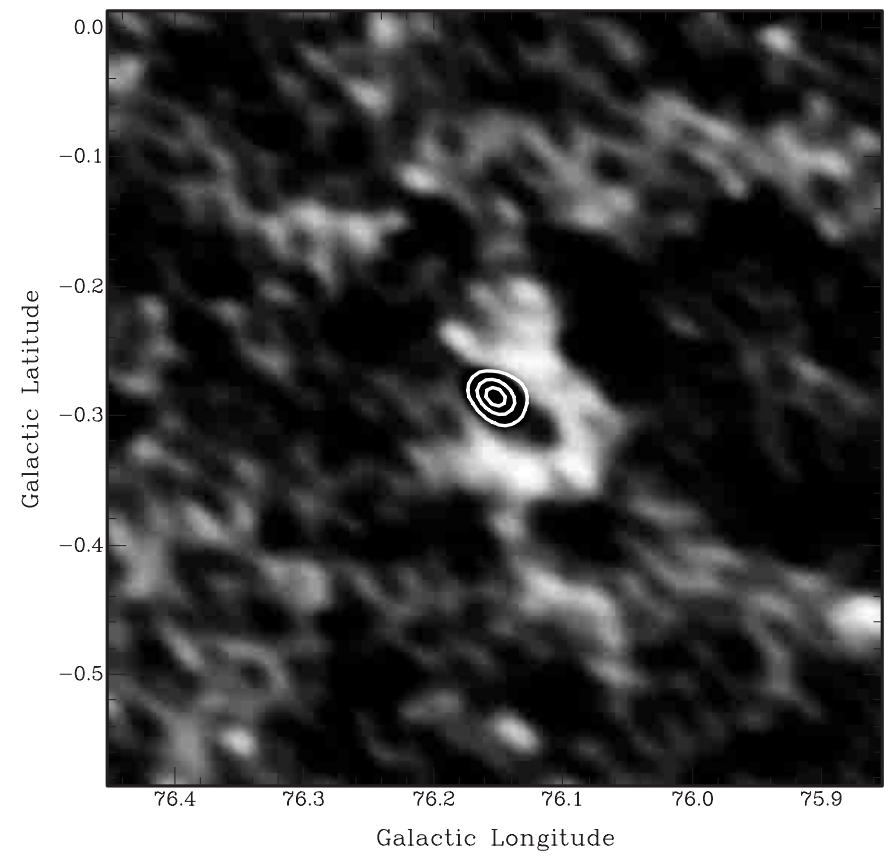

Fig. 10. The $\mathrm{H}_{\text {I }}$ shell found in emission around the $\mathrm{H}_{\text {II }}$ region $\mathrm{G} 76.15$ integrated between -22 and $-30 \mathrm{~km} \mathrm{~s}^{-1}$.

\subsection{Where is the receding shell?}

We identified most of the additional absorption lines as the absorption of the H II region's radio continuum emission by the expanding layer of shocked neutral hydrogen around the ionized gas. Now we have to discuss the receding parts of these symmetric layers. By utilizing Eqs. (1) and (2) we can calculate the amplitude ratio of the absorption line of the approaching shell to the emission line of the receding shell, which results in

$\left|\frac{T_{\mathrm{B}}\left(v_{\mathrm{d}}-v_{\mathrm{exp}}\right)}{T_{\mathrm{B}}\left(v_{\mathrm{d}}+v_{\mathrm{exp}}\right)}\right|=\frac{\left|T_{\mathrm{S}}-T_{\mathrm{C}}\right|}{T_{\mathrm{S}}}$.

This ratio would be 1 if the radio continuum temperature is twice the spin temperature. For the compact $\mathrm{H}_{\text {II }}$ regions we should expect radio continuum brightness temperatures of up to $1000 \mathrm{~K}$, maybe even $2000 \mathrm{~K}$, at $1420 \mathrm{MHz}$, while the spin temperature should be around a few $100 \mathrm{~K}$ up to a maximum of $1000 \mathrm{~K}$. Since most sources show rather complex foreground absorption spectra it is very difficult to decide if a structure is an emission line superposed on the foreground absorption or just the lack of absorption at this position. In four cases, G76.15, G76.19, G93.86, and G114.07, we may see the receding shell in emission. All of these sources show a possible line at about the expected radial velocity $v_{\mathrm{d}}+v_{\text {exp }}$. We get amplitude ratios between the absorption line of the approaching shell and the emission line of the receding shell of about 3:1 for G76.15 and G114.07 and about 3:2 for G76.19 and G93.86. This would translate to $T_{\mathrm{c}}$ to $T_{\mathrm{s}}$ ratios of 4 and 2.5 respectively.

For those objects where we do not see the emission line one can calculate the $T_{\mathrm{c}}$ to $T_{\mathrm{s}}$ ratio required to make the line undetectable. The highest $T_{\mathrm{c}}$ to $T_{\mathrm{s}}$ ratios we derive are for G80.35 and G85.25. These sources show neither emission nor absorption at the expected radial velocity for the receding shell and we need ratios of around 6 so that the amplitude of the receding shell is hidden in the noise. This is not a problem for the typical values for $T_{\mathrm{c}}$ and $T_{\mathrm{s}}$ we discussed above.

\subsection{G76.15-0.29, a special case?}

For none of the sources in our sample we would expect to observe either of the neutral layers around them in emission because these sources are unresolved and thus this emission should be buried within the absorption lines. Especially the thin shocked layer should be impossible to detect in our data. However, around the source G76.15, which is slightly extended in our data, we found an incomplete shell of $\mathrm{H}$ I emission centered on its recombination line velocity (see Fig. 10). This H I shell has a $u$-shaped structure open to the north-east. In the north-west and the south-east it seems to be located right outside the $\mathrm{H}_{\text {II }}$ region while there is a gap between them in the south-west. We derived emission profiles for this structures at different radii from the center of the $\mathrm{H}$ II region going to the north-west (see Fig. 11). The separation of the two emission peaks indicate an expansion velocity of $5-6 \mathrm{~km} \mathrm{~s}^{-1}$. The existence of the emission gap between the $\mathrm{H}_{\text {II }}$ region and the $\mathrm{H}_{\text {I }}$ shell in the south-west demonstrates that the H I shell cannot be the layer of shocked neutral hydrogen, since this layer should be located right outside the ionization front. Hence, this expanding shell must be the layer of unshocked dissociated molecular material. The only explanation for the systematic velocity difference of $5-6 \mathrm{~km} \mathrm{~s}^{-1}$ is that the $\mathrm{H}$ II region is not completely surrounded by molecular material. Thus we can expect flows of neutral hydrogen towards low density areas giving the material a velocity along the line of sight. G76.15 must also be a blister $\mathrm{H}$ II region.

\section{Summary}

We developed a background filtering technique that allows the detection of small-scale positive and negative features within a data set. The filtering was applied to CGPS $\mathrm{H}_{\mathrm{I}}$ observations towards 26 compact $\mathrm{H}$ II regions in order to obtain absorption and emission spectra.

In all but three of the spectra we observe absorption lines at more negative velocities than would be expected from the absorption of continuum emission by foreground material. A simple model of an expanding $\mathrm{H}_{\text {II }}$ region with surrounding shocked and unshocked $\mathrm{H}_{\mathrm{I}}$ zones was developed to interpret the observations. For the majority of the observations, the physical properties (expansion velocity, column density) of the shells derived from the absorption spectra are consistent with them being caused by shocked expanding shells of $\mathrm{H}$.

Some of the additional lines we observe are caused by a large scale Galactic shock associated with the Perseus arm of the Galaxy. Evidence for this comes from the way in which the velocity of the observed absorption features vary with longitude.

Finally, two of the observed $\mathrm{H}$ in regions show evidence for high velocity neutral flows such as the flows observed in emission towards DR 21. 

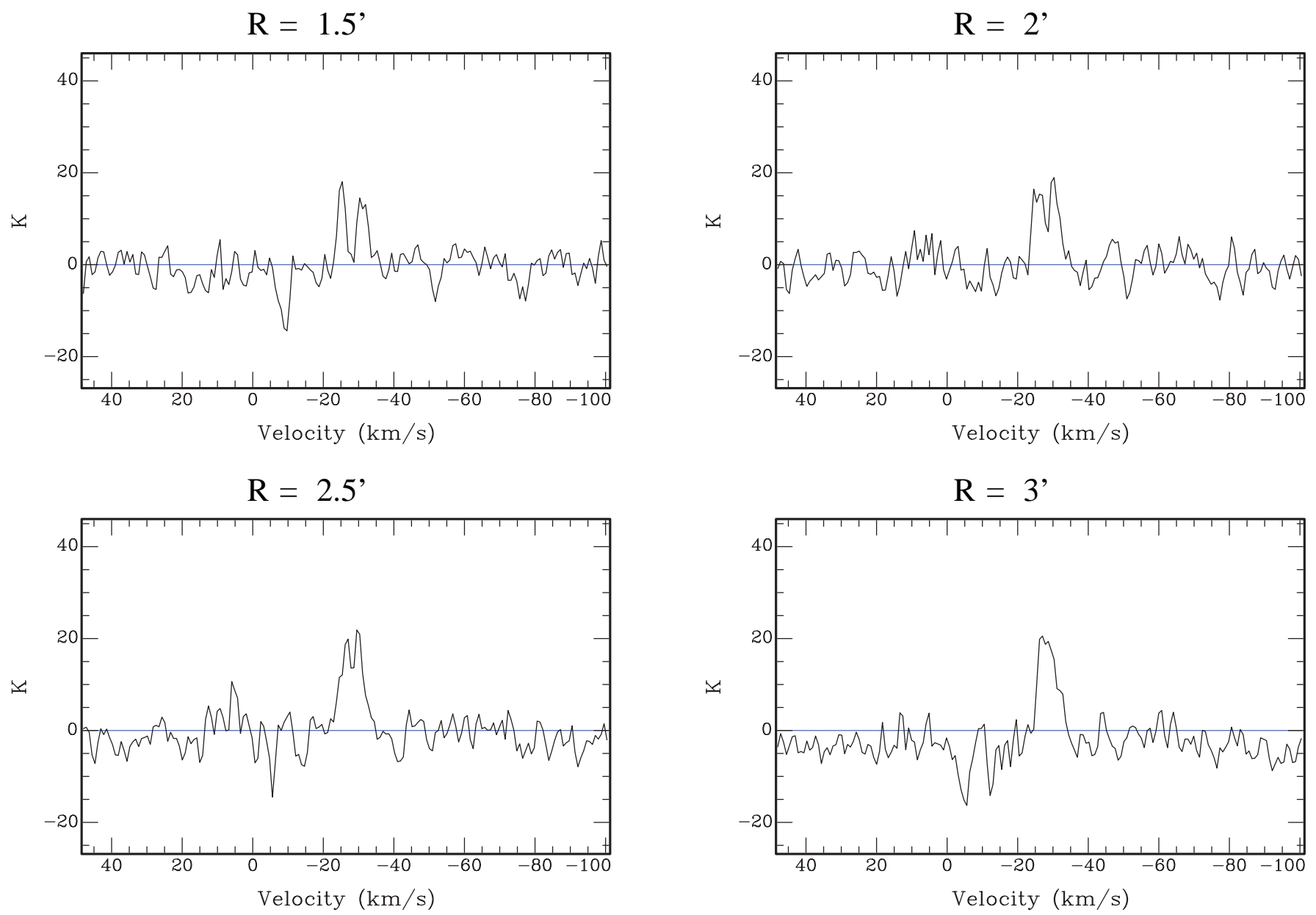

Fig. 11. Emission profiles of the $\mathrm{H}_{\mathrm{I}}$ shell around G76.15 taken at different radii.

This study has shown how absorption spectra can be used to examine neutral material surrounding $\mathrm{H}$ ir regions even if the $\mathrm{H}$ II regions are unresolved. The techniques that have been developed should prove to be useful as radio observations of the Galactic plane at $1^{\prime}$ resolution are extended beyond the CGPS region to cover the majority of the plane.

Acknowledgements. The Dominion Radio Astrophysical Observatory is a National Facility operated by the National Research Council. The Canadian Galactic Plane Survey is a Canadian project with international partners, and is supported by the Natural Sciences and Engineering Research Council (NSERC).

\section{References}

Brand, J., \& Blitz, L. 1993, A\&A, 275, 67

Brunt, C. M., \& Kerton, C. 2002, ApJ, submitted

Bronfman, L., Nyman, L.-A., \& May, J. 1996, A\&AS, 115, 81

Dewdney, P. E., \& Roger, R. S. 1982, ApJ, 235, 564

Franco, J., Tenorio-Tagle, G., \& Bodenheimer, P. 1989, RMxAA, 18, 65
Garay, G., Rodriguez, L. F., Moran, J. M., \& Churchwell, E. 1993, ApJ, 418, 368

Gómez, Y., Lebron, M., Rodriguez, L. F., et al. 1998, ApJ, 503, 297

Higgs, L. A. 2000, in Imaging at Radio through Submillimeter Wavelengths, ed. J. G. Mangum, \& S. J. E. Radford, ASP Conf. Ser., 217, 259

Hill, J. K., \& Hollenbach, D. J. 1978, ApJ, 225, 390

Kuchar, T. A., \& Bania, T. M. 1990, ApJ, 352, 192

Kuchar, T. A., \& Bania, T. M. 1994, ApJ, 436, 117

Kurtz, S., Churchwell, E., \& Wood, D. O. S. 1994, ApJS, 91, 659

Landecker, T. L., et al. 2000, A\&AS, 145, 509

Lebrón, M. E., Rodriguez, L. F., \& Lizano, S. 2001, ApJ, 560, 806

Lockman, F. J. 1989, ApJS, 71, 469

McClure-Griffiths, N. M., et al. 2001, ApJ, 551, 394

Normandeau, M. 1999, AJ, 117, 2440

Richards, P. J., Little, L. T., Toriseva, M., \& Heaton, B. D. 1987, MNRAS, 228, 43

Roberts, W. W. 1972, ApJ, 173, 259

Roberts, D. A., Dickel, H. R., \& Goss, W. M. 1997, ApJ, 476, 209

Roger, R. S. 1982, in Regions of Recent Star Formation, ed. R. S. Roger, \& P. E. Dewdney (Dordrecht, Reidel), 167

Roger, R. S., \& Dewdney, P. E. 1992, ApJ, 385, 536

Roger, R. S., \& Irwin, J. A. 1982, ApJ, 256, 127

Roger, R. S., \& Pedlar, A. 1981, A\&A, 94, 238 
Russell, A. P. G., Bally, J., Padman, R., \& Hills, R. E. 1992, ApJ, 387, van den Ancker, M. E., Tielens, A. G. G. M., \& Wesselius, P. R. 2000, 219

Shepherd, D. S., \& Churchwell, E. ApJ, 457, 267

Sofue, Y., \& Reich, W. 1979, A\&AS, 38, 251

Taylor, A. R. 1999, in New Perspectives on the Interstellar Medium, ed. A. R. Taylor, T. L. Landecker, \& G. Joncas, ASP Conf. Ser., 168,3

A\&A, 358, 1035

van def Werf, P. P., \& Goss, W. M. 1989, A\&A, 224, 209

van def Werf, P. P., \& Goss, W. M. 1990, A\&A, 238, 296

Wendker, H. J., \& Wrigge, M. 1996, A\&A, 305, 592

Wood, D. O. S., \& Churchwell, E. 1988, ApJS, 69, 831

Wouterloot, J. G. A., \& Brand, J. 1993, A\&AS, 80, 149

Timmermann, R., Bertoldi, F., Wright, C. M., et al. 1996, A\&A, 315, L281 